\title{
A23 MYOSITIS ASSOCIATED AUTOANTIBODIES DETECTED USING A NOVEL RECOMBINANT PROTEIN BLOTTING: CLINICAL ASSOCIATIONS
}

P J Charles, I E Lundberg, E Paterson, L Ekholm, K Dankó, I Putova, J Vencovsky Kennedy Institute, UK

\subsection{6/ard.2010.129577w}

Purpose Screening for autoantibodies associated with idiopathic inflammatory myopathies (IIM) has conventionally been performed using radio-immunoprecipitation techniques which are not suitable for use in routine diagnostic laboratories. The authors have therefore investigated the use of lineimmunoassay technology employing recombinant antigens as an alternative for this purpose.

Methods Sera and clinical data were collected on 486 patients (256 PM,183 DM, 20 JDM, 9 IBM, 18 overlap syndrome) with IIM, according to Bohan and Peter or Grigg's criteria, from three European `s as part of the EUMyogen study. These were tested using a line-immunoassay system (Euroimmun, Lubeck, Germany). The system tested was able to detect 10 myositis associated antibodies (Jo-1, PL-7, PL-12, EJ, OJ, SRP, PM-Scl, $\mathrm{Ku}, \mathrm{Mi}-2 \beta, \mathrm{U} 1-\mathrm{RNP})$. Patients were assesed for the presence of joint disease, interstitial lung disease (ILD), Gottron's papules, heliotrope rash and cancer( \pm 3 years of diagnosis).

Results Anti Jo-1 was the commonest antibody detected in the cohort (12.6\%). Antibodies to PM-Scl (3.7\%); U1-RNP (5.5\%); SRP (2.6\%); Mi-2ß (2.6\%); Ku (1.6\%); EJ, PL-7; and PL-12 (all $<1 \%$ ) were also detected in the cohort.

Anti-Jo-1 ( $p=0.0001 ;$ OR10.25). Anti-PM-Scl ( $p=0.04 ;$ OR 5.8) and Anti-SRP ( $p=0.04$; OR 5.6) were associated with the presence of ILD. Anti-Mi-2 $\beta$ ( $p=0.001$; OR 12.57) was associated with the presence of Gottron's papules. Antibodies 
to PM-Scl were significantly associated with the presence of scleroderma overlap ( $p=0.014$; OR 11.9). Anti-Jo-1 and Anti-U1 RNP were negatively associated with presence of Gottron's papules and heliotrope rash. None of the antibody specificities tested were associated with joint disease or cancer.

Conclusion In this study, using recombinant protein lineimmunoassay technology, the authors have reported that antibodies to Jo-1, SRP and PM-Scl are associated with the presence of ILD, and that antibodies to $\mathrm{Mi}-2 \beta$ are associated with the presence of Gottron's papules. In addition antibodies to Jo-1 and U1 RNP show a negative association with the presence of Gottron's papules and heliotrope rash. Detection of myositis associated antibodies using line-immunoassay technology employing recombinant protein antigens is a fast and reporducible method, which is easy to perform, and which will allow the assaying of these clinically significant antibodies in routine clinical laboratories

infections. 